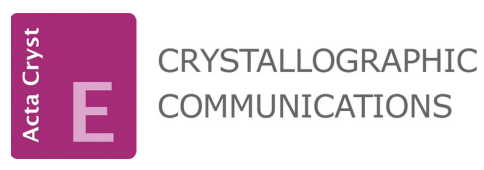

ISSN 2056-9890

Received 21 May 2020

Accepted 19 June 2020

Edited by S. Parkin, University of Kentucky, USA

Keywords: crystal structure; salicylaldehyde derivative; 4-iodoaniline; 2-hydroxy-5-nitrobenzaldehyde; hydrogen bonding.

CCDC reference: 1922980

Supporting information: this article has supporting information at journals.iucr.org/e

\section{Crystal structure and Hirshfeld surface analysis of 2-\{[(4-iodophenyl)imino]methyl\}-4-nitrophenol}

\author{
Md. Serajul Haque Faizi, ${ }^{\text {a }}$ Tenzile Alagöz, ${ }^{\mathrm{b}}$ Ruby Ahmed, ${ }^{\mathrm{c}}$ Emine Berrin Cinar, \\ Erbil Agar, ${ }^{b}$ Necmi Dege ${ }^{d}$ and Ashraf Mashrai ${ }^{e_{*}}$
}

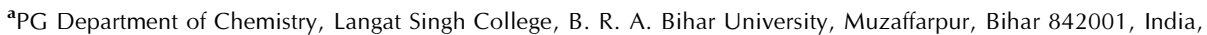
${ }^{\mathbf{b}}$ Ondokuz Mayıs University, Faculty of Arts and Sciences, Department of Chemistry, Samsun, Turkey, ${ }^{\mathbf{c} D e p a r t m e n t}$ of Applied Chemistry, ZHCET, Aligarh Muslim University, Aligarh, 202002, UP, India, ' Ondokuz Mayıs University, Faculty of Arts and Sciences, Department of Physics, Samsun, Turkey, and ${ }^{\mathbf{e}}$ Department of Pharmacy, University of Science and Technology, Ibb Branch, Ibb, Yemen. *Correspondence e-mail: ashraf.yemen7@gmail.com, ashraf.yemen7@gmail.com

The title compound, $\mathrm{C}_{13} \mathrm{H}_{9} \mathrm{IN}_{2} \mathrm{O}_{3}$, was synthesized by a condensation reaction between 2-hydroxy-5-nitrobenzaldehyde and 4-iodoaniline, and crystallizes in the orthorhombic space group $P n a 2_{1}$. The 4-iodobenzene ring is inclined to the phenol ring by a dihedral angle of 39.1 (2) $)^{\circ}$ The configuration about the $\mathrm{C}=\mathrm{N}$ double bond is $E$. The crystal structure features $\mathrm{C}-\mathrm{H} \cdots \mathrm{O}$ hydrogen-bonding interactions. A Hirshfeld surface analysis of the crystal structure indicates that the most important contributions for the packing arrangement are $\mathrm{O} \cdots \mathrm{H} /$ $\mathrm{H} \cdot \mathrm{O}(26.9 \%)$ and $\mathrm{H} \cdot \mathrm{H}(22.0 \%)$ interactions.

\section{Chemical context}

Over the past 25 years, extensive research has been directed towards the synthesis and use of Schiff base compounds in organic and inorganic chemistry as they have important medicinal and pharmaceutical applications. These compounds exhibit biological activities, including antibacterial, antifungal, anticancer and herbicidal properties (Desai et al., 2001; Singh \& Dash, 1988; Karia \& Parsania, 1999). They may also show useful photochromic properties, leading to applications in various fields such as the measurement and control of radiation intensities in imaging systems and optical computers, electronics, optoelectronics and photonics (Iwan et al., 2007). Schiff bases derived from 2-hydroxy-5-nitrobenzaldehyde are widely used either as materials or as intermediates in explosives, dyestuffs, pesticides and organic synthesis (Yan et al., 2006). Intramolecular hydrogen-atom transfer (tautomerism) from the $o$-hydroxy group to the imine- $\mathrm{N}$ atom is of prime importance with respect to the solvato-, thermo- and photochromic properties of $o$-hydroxy Schiff bases (Filarowski, 2005; Hadjoudis \& Mavridis 2004). Such proton-exchanging materials can be utilized for the design of various molecular electronic devices (Alarcón et al., 1999). The present work is a part of an ongoing structural study of Schiff bases and their utilization in the synthesis of quinoxaline derivatives (Faizi et al., 2018), fluorescence sensors (Faizi et al., 2016; Mukherjee et al., 2018; Kumar et al., 2017; 2018) and non-linear optical properties (Faizi et al., 2020). We report herein the synthesis (from 2-hydroxy-5-nitrobenzaldehyde and 4-iodoaniline) and crystal structure of the title compound (I), along with the findings of a Hirshfeld surface analysis. 
<smiles>O=[N+]([O-])c1ccc(O)c(/C=N/c2ccc(I)cc2)c1</smiles>

\section{Structural commentary}

The molecular structure of compound (I) is shown in Fig. 1. An intramolecular $\mathrm{O}-\mathrm{H} \cdots \mathrm{N}$ hydrogen bond is observed (Table 1 and Fig. 1). This is a relatively common feature in analogous imine-phenol compounds (see Database survey section). The imine group displays a $\mathrm{C} 8-\mathrm{C} 7-\mathrm{N} 1-\mathrm{C} 4$ torsion angle of $174.5(6)^{\circ}$. The 4-iodobenzene ring $(\mathrm{C} 1-\mathrm{C} 6)$ is inclined by a dihedral angle of $39.1(2)^{\circ}$ to the phenol ring (C8-C13), which renders the molecule non-planar. The configuration of the $\mathrm{C} 7=\mathrm{N} 1$ bond of this Schiff base is $E$, and the intramolecular $\mathrm{O} 1-\mathrm{H} 1 \cdots \mathrm{N} 1$ hydrogen bond forms an $S(6)$ ring motif (Fig. 1 and Table 1). The 4-nitro group is slightly tilted away from co-planarity with the benzene ring to which it is attached $\left[\mathrm{O} 2-\mathrm{N} 2-\mathrm{C} 10-\mathrm{C} 9=-7.4(10)^{\circ}\right.$ and $\left.\mathrm{O} 3-\mathrm{N} 2-\mathrm{C} 10-\mathrm{C} 11=-7.4(10)^{\circ}\right]$. The $\mathrm{C} 13-\mathrm{O} 1$ distance [1.330 (7) $\AA$ ] is close to normal for values reported for single $\mathrm{C}-\mathrm{O}$ bonds in phenols and salicylideneamines (Ozeryanskii et al., 2006). The $\mathrm{N} 1=\mathrm{C} 7$ bond is short at 1.264 (8) $\AA$, indicative of double-bond character, while the long $\mathrm{C} 7-\mathrm{C} 8$ bond [1.444 (8) $\AA$ ] implies a single bond. All these data support the existence of the phenol-imine tautomer for (I) in its crystalline state. These features are similar to those observed in related 4-dimethylamino- $N$-salicylideneanilines (Filipenko et al., 1983; Aldoshin et al., 1984; Wozniak et al., 1995; Pizzala et al., 2000). The $\mathrm{C}-\mathrm{N}, \mathrm{C}=\mathrm{N}$ and $\mathrm{C}-\mathrm{C}$ bond lengths are normal and close to the values observed in related structures (Faizi $e t$ al., 2017a,b).

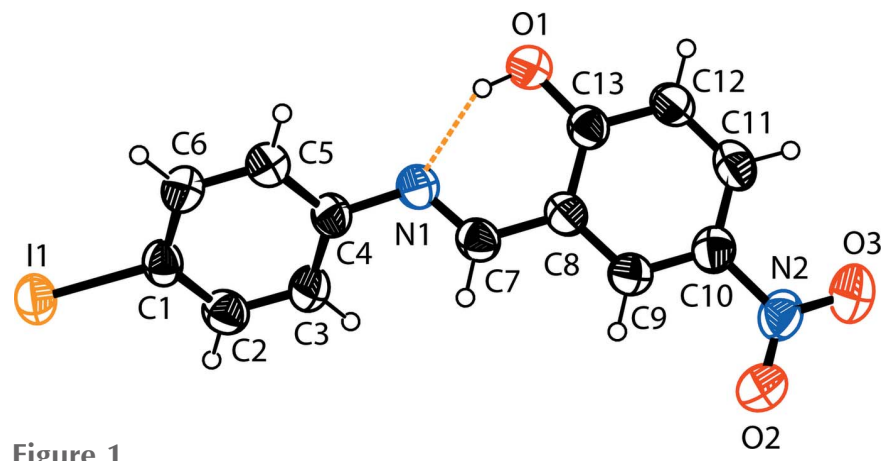

Figure 1

The molecular structure of the title compound, with the atom labelling. Displacement ellipsoids are drawn at the $40 \%$ probability level. The intramolecular $\mathrm{N}-\mathrm{H} \cdots \mathrm{O}$ hydrogen bond (see Table1), forming an $S(6)$ ring motif, is shown as a dashed line.
Table 1

Hydrogen-bond geometry $\left(\AA{ }^{\circ}\right)$.

\begin{tabular}{lllll}
\hline$D-\mathrm{H} \cdots A$ & $D-\mathrm{H}$ & $\mathrm{H} \cdots A$ & $D \cdots A$ & $D-\mathrm{H} \cdots A$ \\
\hline $\mathrm{O} 1-\mathrm{H} 1 \cdots \mathrm{N} 1$ & 0.82 & 1.86 & $2.591(6)$ & 148 \\
$\mathrm{C} 7-\mathrm{H} 7 \cdots \mathrm{O} 2^{\mathrm{i}}$ & 0.93 & 2.45 & $3.309(8)$ & 154 \\
\hline
\end{tabular}

Symmetry code: (i) $-x+1,-y+1, z-\frac{1}{2}$.

\section{Supramolecular features}

In the crystal packing of (I), the most important intermolecular interactions are weak $\mathrm{C} 7-\mathrm{H} 7 \cdots \mathrm{O} 2^{\mathrm{i}}$ [symmetry code: (i) $1-x, 1-y,-\frac{1}{2}+z$ ] hydrogen bonds between screwrelated molecules, which form helical chains propagating along the crystallographic screw axis parallel to $c$ (Fig. 2, Table 1). The shortest intermolecular contact involving the iodine is $3.351(5) \AA$, between glide-related molecules, I1 $\cdots \mathrm{O} 1^{\mathrm{ii}}$ [symmetry code: (ii) $x+\frac{1}{2}, \frac{1}{2}-y,-1+z$ )], which makes a zigzag tape motif (Fig. 3). There are no other

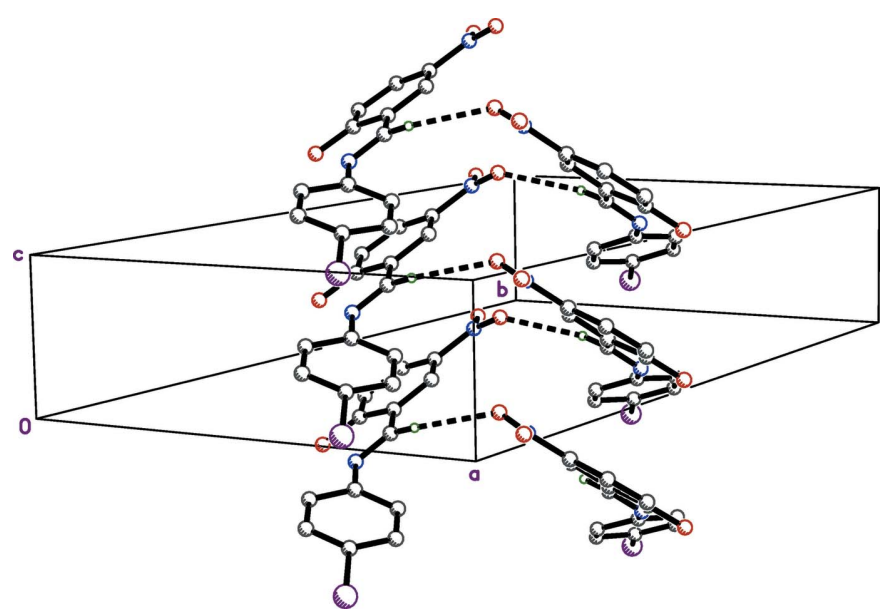

Figure 2

A partial packing plot showing the $\mathrm{C}-\mathrm{H} \cdots \mathrm{O}$ hydrogen-bonded (thick dashed lines) helical chains about the crystallographic $2_{1}$ screw axis parallel to $c$.

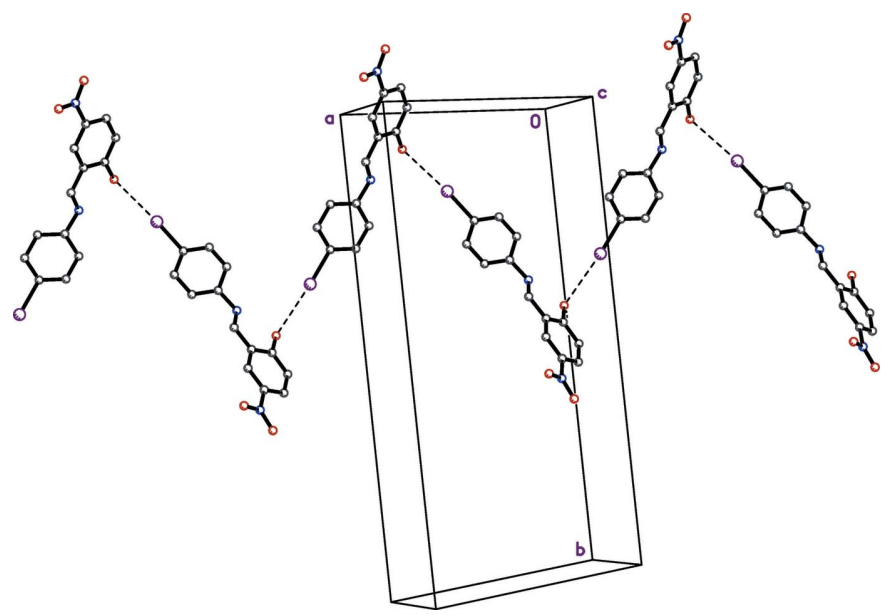

Figure 3

A partial packing plot showing close contacts (dashed lines) between iodine and the phenolic oxygen of glide-related $\left(x+\frac{1}{2}, \frac{1}{2}-y,-1+z\right)$ molecules. 
significant intermolecular interactions present in the crystal. The Hirshfeld surface analysis confirms the role of the $\mathrm{C}-$ $\mathrm{H} \cdots \mathrm{O}$ interactions in the packing arrangement.

\section{Hirshfeld surface analysis}

In order to visualize the intermolecular interactions in the crystal packing of (I), a Hirshfeld surface (HS) analysis (Hirshfeld, 1977; Spackman \& Jayatilaka, 2009) was carried out using Crystal Explorer 17.5 (Turner et al., 2017). In the HS plotted over $d_{\text {norm }}$ (Fig. 4), white surfaces indicate contacts with distances equal to the sum of van der Waals radii, and the red and blue colours indicate distances shorter (i.e., in close contact) or longer than the van der Waals radii sum, respectively (Venkatesan et al., 2016). The two-dimensional finger print plots are depicted in Fig. 5. The O $\cdots \mathrm{H} / \mathrm{H} \cdots \mathrm{O}(26.9 \%)$ interactions form the majority of contacts, with $\mathrm{H} \cdots \mathrm{H}$ $(22.0 \%)$ interactions representing the next highest contribution. The percentage contributions of other interactions are: $\mathrm{I} \cdots \mathrm{H} / \mathrm{H} \cdots \mathrm{I}(16.3 \%), \mathrm{C} \cdots \mathrm{H} / \mathrm{H} \cdots \mathrm{C}(10.5 \%), \mathrm{C} \cdots \mathrm{C}(8.7 \%)$, $\mathrm{O} \cdots \mathrm{C} / \mathrm{C} \cdots \mathrm{O} \quad(4.7 \%), \quad \mathrm{N} \cdots \mathrm{C} / \mathrm{C} \cdots \mathrm{N} \quad(3.8 \%), \quad \mathrm{I} \cdots \mathrm{C} / \mathrm{C} \cdots \mathrm{I}$ $(2.3 \%), \mathrm{H} \cdots \mathrm{N} / \mathrm{N} \cdots \mathrm{H}(1.4 \%), \mathrm{I} \cdots \mathrm{O} / \mathrm{O} \cdots \mathrm{I} \quad(2.0 \%), \mathrm{I} \cdots \mathrm{N} /$ $\mathrm{N} \cdots \mathrm{I}(0.6 \%), \mathrm{I} \cdots \mathrm{I}(0.5 \%), \mathrm{O} \cdots \mathrm{N} / \mathrm{N} \cdots \mathrm{O} \quad(0.2 \%), \mathrm{N} \cdots \mathrm{N}$ $(0.1 \%)$ and $\mathrm{O} \cdots \mathrm{O}(0.1 \%)$.

\section{Database survey}

A search of the Cambridge Structural Database (CSD, version 5.39; Groom et al., 2016) gave 26 hits for the (E)-2-\{[(4-iodophenyl)imino]methyl\}-phenol fragment. Of these 26, the most similar to (I), are as follows. In $p$-iodo- $N$ - $(p$-cyanobenzylidene)aniline (LALMEQ; Ojala et al., 1999), the OH group is absent and the $\mathrm{NO}_{2}$ group is replaced by a cyano group. In (E)-5-(diethylamino)-2-[(4-iodophenylimino)methyl]phenol (VEFPED; Kaştaş et al., 2012), the $\mathrm{NO}_{2}$ is replaced by an $N, N$ diethyl group. In $N$-(3,5-di-tert-butylsalicylidene)-4-iodobenzene; (MILFET; Spangenberg et al., 2007), the $\mathrm{NO}_{2}$ group is absent but a pair of ${ }^{t} \mathrm{Bu}$ groups occupy the 3,5 positions of the salicylidene group. In 2-\{[(4-iodophenyl)imino]methyl\}-6-

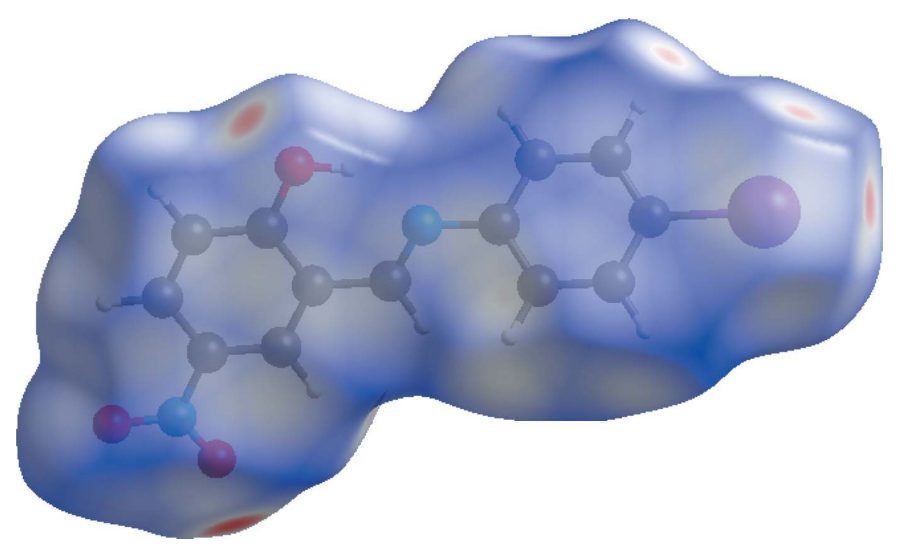

Figure 4

Hirshfeld surface of the title compound plotted over $d_{\text {norm }}$.
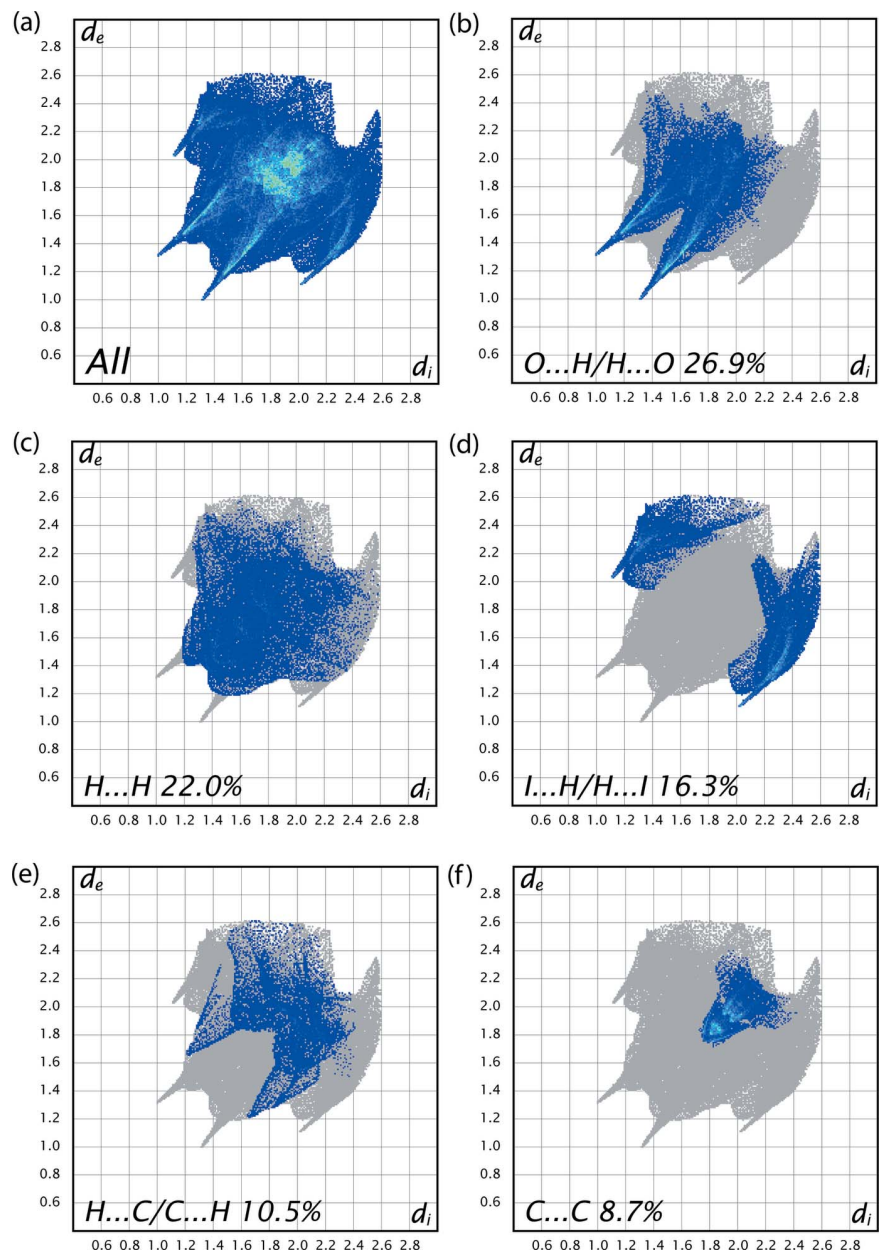

Figure 5

Two-dimensional fingerprint plots of the crystal with the relative contributions of the atom pairs to the Hirshfeld surface along with $d_{\text {norm }}$ full.

methoxyphenol (SEDBIP; Carletta, et al., 2017), the $\mathrm{NO}_{2}$ group is absent and a methoxy group is ortho to the hydroxyl. Lastly, in $\mathrm{N}$-(2-cyanobenzylidene)-4-iodoaniline (XOXKIF; Ojala et al., 1999) the $\mathrm{NO}_{2}$ is absent and the $\mathrm{OH}$ is replaced by cyano. All these compounds have an $E$ configuration about the $\mathrm{C}=\mathrm{N}$ bond and form the $S(6)$ ring motif.

\section{Synthesis and crystallization}

The title compound was synthesized by condensation of 2-hydroxy-5-nitrobenzaldehyde $(11.0 \mathrm{mg}, 0.066 \mathrm{mmol})$ and 4-iodoaniline $(14.4 \mathrm{mg}, 0.066 \mathrm{mmol})$ in ethanol $(15 \mathrm{ml})$. After the mixture had refluxed for about $15 \mathrm{~h}$, the orange product was washed with ether and dried at room temperature (yield $60 \%$, m.p. 484-486 K). Crystals suitable for X-ray analysis were obtained by slow evaporation of an ethanol solution.

\section{Refinement}

Crystal data, data collection and structure refinement details are summarized in Table 2. The $\mathrm{OH}$ hydrogen atoms and the 
Table 2

Experimental details.

\begin{tabular}{|c|c|}
\hline \multicolumn{2}{|l|}{ Crystal data } \\
\hline Chemical formula & $\mathrm{C}_{13} \mathrm{H}_{9} \mathrm{IN}_{2} \mathrm{O}_{3}$ \\
\hline$M_{\mathrm{r}}$ & 368.12 \\
\hline Crystal system, space group & Orthorhombic, Pna2 $2_{1}$ \\
\hline Temperature $(\mathrm{K})$ & 296 \\
\hline$a, b, c(\AA)$ & $12.8022(4), 24.4556(9), 4.1459$ (1) \\
\hline$V\left(\AA^{3}\right)$ & $1298.02(7)$ \\
\hline$Z$ & 4 \\
\hline Radiation type & Мо $K \alpha$ \\
\hline$\mu\left(\mathrm{mm}^{-1}\right)$ & 2.47 \\
\hline Crystal size $(\mathrm{mm})$ & $0.42 \times 0.34 \times 0.21$ \\
\hline \multicolumn{2}{|l|}{ Data collection } \\
\hline Diffractometer & Stoe IPDS 2 \\
\hline Absorption correction & $\begin{array}{l}\text { Integration ( } X \text {-RED32; Stoe \& Cie, } \\
\text { 2002) }\end{array}$ \\
\hline$T_{\min }, T_{\max }$ & $0.944,0.981$ \\
\hline $\begin{array}{l}\text { No. of measured, independent and } \\
\text { observed }[I>2 \sigma(I)] \text { reflections }\end{array}$ & $15403,2508,2231$ \\
\hline$R_{\text {int }}$ & 0.084 \\
\hline$(\sin \theta / \lambda)_{\max }\left(\AA^{-1}\right)$ & 0.617 \\
\hline \multicolumn{2}{|l|}{ Refinement } \\
\hline$R\left[F^{2}>2 \sigma\left(F^{2}\right)\right], w R\left(F^{2}\right), S$ & $0.037,0.094,1.05$ \\
\hline No. of reflections & 2508 \\
\hline No. of parameters & 173 \\
\hline No. of restraints & 1 \\
\hline $\mathrm{H}$-atom treatment & H-atom parameters constrained \\
\hline$\Delta \rho_{\max }, \Delta \rho_{\min }\left(\mathrm{e} \AA^{-3}\right)$ & $0.81,-0.25$ \\
\hline Absolute structure & $\begin{array}{l}\text { Flack } x \text { determined using } 814 \\
\quad \text { quotients }\left[\left(I^{+}\right)-\left(I^{-}\right)\right] /\left[\left(I^{+}\right)+\left(I^{-}\right)\right] \\
\quad \text { (Parsons } \text { et al., 2013) }\end{array}$ \\
\hline Absolute structure parameter & $0.00(4)$ \\
\hline
\end{tabular}

Computer programs: X-AREA and X-SHAPE (Stoe \& Cie, 2002), SHELXT2018/2 (Sheldrick, 2015a), SHELXL2018/3 (Sheldrick, 2015b), ORTEP-3 for Windows (Farrugia, 2012) and XP in SHELXTL (Sheldrick, 2008).

C-bound $\mathrm{H}$ atoms were included in calculated positions and allowed to ride on the parent atoms: $\mathrm{O}-\mathrm{H}=0.82 \AA, \mathrm{C}-\mathrm{H}=$ $0.93-0.96 \AA$ with $U_{\text {iso }}(\mathrm{H})=1.5 U_{\text {eq }}(\mathrm{C}$-methyl $)$ and $1.2 U_{\text {eq }}(\mathrm{C})$ for other $\mathrm{H}$ atoms.

\section{Acknowledgements}

The authors are grateful to the Department of Chemistry, Langat Singh College, B. R. A. Bihar University, Muzaffarpur, India, for providing laboratory facilities.

\section{Funding information}

The authors thank the Faculty of Pharmacy, University of Science and Technology, Ibb Branch, Ibb, Yemen for financial support. Funding for this research was provided by astart-up grant from the University Grants Commission (India).

\section{References}

Alarcón, S. H., Pagani, D., Bacigalupo, J. \& Olivieri, A. C. (1999). J. Mol. Struct. 475, 233-240.

Aldoshin, S. M., Atovmyan, L. O. \& Ponomarev, V. I. (1984). Khim. Fiz. 3, 787-791.
Carletta, A., Spinelli, F., d'Agostino, S., Ventura, B., Chierotti, M. R., Gobetto, R., Wouters, J. \& Grepioni, F. (2017). Chem. Eur. J. 23, 5317-5329.

Desai, S. B., Desai, P. B. \& Desai, K. R. (2001). Heterocycl. Commun. 7, 83-90.

Faizi, M. S. H., Ahmad, M., Kapshuk, A. A. \& Golenya, I. A. (2017a). Acta Cryst. E73, 38-40.

Faizi, M. S. H., Alam, M. J., Haque, A., Ahmad, S., Shahid, M. \& Ahmad, M. (2018). J. Mol. Struct. 1156, 457-464.

Faizi, M. S. H., Dege, N., Haque, A., Kalibabchuk, V. A. \& Cemberci, M. (2017b). Acta Cryst. E73, 96-98.

Faizi, M. S. H., Gupta, S., Mohan, V. K., Jain, K. V. \& Sen, P. (2016). Sens. Actuators B Chem. 222, 15-20.

Faizi, M. S. H., Osório, F. A. P. \& Valverde, C. (2020). J. Mol. Struct. 1210, 128039-464.

Farrugia, L. J. (2012). J. Appl. Cryst. 45, 849-854.

Filarowski, A. (2005). J. Phys. Org. Chem. 18, 686-698.

Filipenko, O. S., Ponomarev, V. I., Bolotin, B. M. \& Atovmyan, L. O. (1983). Kristallografiya, 28, 889-895.

Groom, C. R., Bruno, I. J., Lightfoot, M. P. \& Ward, S. C. (2016). Acta Cryst. B72, 171-179.

Hadjoudis, E. \& Mavridis, I. M. (2004). Chem. Soc. Rev. 33, 579-588.

Hirshfeld, H. L. (1977). Theor. Chim. Acta, 44, 129-138.

Iwan, A., Kaczmarczyk, B., Janeczek, H., Sek, D. \& Ostrowski, S. (2007). Spectrochim. Acta A Mol. Biomol. Spectrosc. 66, 1030-1041.

Karia, F. D. \& Parsania, P. H. (1999). Asian J. Chem. 11, 991-995.

Kaştaş, G., Albayrak, C., Odabaşoğlu, M. \& Frank, R. (2012). Spectrochim. Acta A Mol. Biomol. Spectrosc. 94, 200-204.

Kumar, M., Kumar, A., Faizi, M. S. H., Kumar, S., Singh, M. K., Sahu, S. K., Kishor, S. \& John, R. P. (2018). Sens. Actuators B Chem. 260, 888-899.

Kumar, S., Hansda, A., Chandra, A., Kumar, A., Kumar, M., Sithambaresan, M., Faizi, M. S. H., Kumar, V. \& John, R. P. (2017). Polyhedron, 134, 11-21.

Mukherjee, P., Das, A., Faizi, M. S. H. \& Sen, P. (2018). Chemistry Select, 3, 3787-3796.

Ojala, C. R., Ojala, W. H., Gleason, W. B. \& Britton, D. (1999). J. Chem. Crystallogr. 29, 27-32.

Ozeryanskii, V. A., Pozharskii, A. F., Schilf, W., Kamieński, B., SawkaDobrowolska, W., Sobczyk, L. \& Grech, E. (2006). Eur. J. Org. Chem. pp. 782-790.

Parsons, S., Flack, H. D. \& Wagner, T. (2013). Acta Cryst. B69, 249 259.

Pizzala, H., Carles, M., Stone, W. E. E. \& Thevand, A. (2000). J. Chem. Soc. Perkin Trans. 2, pp. 935-939.

Sheldrick, G. M. (2008). Acta Cryst. A64, 112-122.

Sheldrick, G. M. (2015a). Acta Cryst. A71, 3-8.

Sheldrick, G. M. (2015b). Acta Cryst. C71, 3-8.

Singh, W. M. \& Dash, B. C. (1988). Pesticides, 22, 33-37.

Spackman, M. A. \& Jayatilaka, D. (2009). CrystEngComm, 11, 19-32.

Spangenberg, A., Sliwa, M., Métivier, R., Dagnélie, R., Brosseau, A., Nakatani, K., Pansu, R. \& Malfant, I. (2007). J. Phys. Org. Chem. 20, 992-997.

Stoe \& Cie (2002). X-AREA, X-RED32 and X-SHAPE. Stoe \& Cie GmbH, Darmstadt, Germany.

Turner, M. J., McKinnon, J. J., Wolff, S. K., Grimwood, D. J., Spackman, P. R., Jayatilaka, D. \& Spackman, M. A. (2017). CrystalExplorer17. The University of Western Australia.

Venkatesan, P., Thamotharan, S., Ilangovan, A., Liang, H. \& Sundius, T. (2016). Spectrochim. Acta Part A, 153, 625-636.

Wozniak, K., He, H., Klinowski, J., Jones, W., Dziembowska, T. \& Grech, E. (1995). J. Chem. Soc. Faraday Trans. 91, 7-85.

Yan, X. F., Xiao, H. M., Gong, X. D. \& Ju, X. H. (2006). J. Mol. Struct. Theochem, 764, 141-148. 


\section{supporting information}

Acta Cryst. (2020). E76, 1146-1149 [https://doi.org/10.1107/S2056989020008191]

\section{Crystal structure and Hirshfeld surface analysis of 2-\{[(4-iodophenyl)imino]- methyl\}-4-nitrophenol}

Md. Serajul Haque Faizi, Tenzile Alagöz, Ruby Ahmed, Emine Berrin Cinar, Erbil Agar, Necmi

Dege and Ashraf Mashrai

Computing details

Data collection: $X$-AREA (Stoe \& Cie, 2002); cell refinement: X-AREA (Stoe \& Cie, 2002); data reduction: X-SHAPE (Stoe \& Cie, 2002); program(s) used to solve structure: SHELXT2018/2 (Sheldrick, 2015a); program(s) used to refine structure: SHELXL2018/3 (Sheldrick, 2015b); molecular graphics: ORTEP-3 for Windows (Farrugia, 2012), XP in SHELXTL (Sheldrick, 2008).

2-\{[(4-lodophenyl)imino]methyl\}-4-nitrophenol

Crystal data

$\mathrm{C}_{13} \mathrm{H}_{9} \mathrm{IN}_{2} \mathrm{O}_{3}$

$M_{r}=368.12$

Orthorhombic, Pna2 ${ }_{1}$

$a=12.8022(4) \AA$

$b=24.4556(9) \AA$

$c=4.1459(1) \AA$

$V=1298.02(7) \AA^{3}$

$Z=4$

$F(000)=712$

Data collection

STOE IPDS 2

diffractometer

Radiation source: sealed X-ray tube, $12 \times 0.4$ $\mathrm{mm}$ long-fine focus

Plane graphite monochromator

Detector resolution: 6.67 pixels $\mathrm{mm}^{-1}$

rotation method scans

Absorption correction: integration

(X-RED32; Stoe \& Cie, 2002)

Refinement

Refinement on $F^{2}$

Least-squares matrix: full

$R\left[F^{2}>2 \sigma\left(F^{2}\right)\right]=0.037$

$w R\left(F^{2}\right)=0.094$

$S=1.05$

2508 reflections

173 parameters

1 restraint
$D_{\mathrm{x}}=1.884 \mathrm{Mg} \mathrm{m}^{-3}$

Mo $K \alpha$ radiation, $\lambda=0.71073 \AA$

Cell parameters from 25449 reflections

$\theta=1.7-29.9^{\circ}$

$\mu=2.47 \mathrm{~mm}^{-1}$

$T=296 \mathrm{~K}$

Prism, colorless

$0.42 \times 0.34 \times 0.21 \mathrm{~mm}$

$T_{\min }=0.944, T_{\max }=0.981$

15403 measured reflections

2508 independent reflections

2231 reflections with $I>2 \sigma(I)$

$R_{\text {int }}=0.084$

$\theta_{\max }=26.0^{\circ}, \theta_{\min }=1.8^{\circ}$

$h=-15 \rightarrow 15$

$k=-30 \rightarrow 30$

$l=-5 \rightarrow 4$

Hydrogen site location: inferred from neighbouring sites

$\mathrm{H}$-atom parameters constrained

$w=1 /\left[\sigma^{2}\left(F_{\mathrm{o}}^{2}\right)+(0.0632 P)^{2}\right]$

where $P=\left(F_{\mathrm{o}}^{2}+2 F_{\mathrm{c}}^{2}\right) / 3$

$(\Delta / \sigma)_{\max }<0.001$

$\Delta \rho_{\max }=0.81$ e $\AA^{-3}$

$\Delta \rho_{\text {min }}=-0.25$ e $\AA^{-3}$ 
Absolute structure: Flack $x$ determined using 814 quotients $\left[\left(I^{+}\right)-(I)\right] /\left[\left(I^{+}\right)+\left(I^{-}\right)\right]$(Parsons et al., 2013)

Absolute structure parameter: $0.00(4)$

\section{Special details}

Geometry. All esds (except the esd in the dihedral angle between two 1.s. planes) are estimated using the full covariance matrix. The cell esds are taken into account individually in the estimation of esds in distances, angles and torsion angles; correlations between esds in cell parameters are only used when they are defined by crystal symmetry. An approximate (isotropic) treatment of cell esds is used for estimating esds involving l.s. planes.

Fractional atomic coordinates and isotropic or equivalent isotropic displacement parameters $\left(\AA^{2}\right)$

\begin{tabular}{lllll}
\hline & $x$ & $y$ & $z$ & $U_{\text {iso }} / U_{\mathrm{eq}}$ \\
\hline I1 & $0.50544(3)$ & $0.16672(2)$ & $-0.1482(5)$ & $0.06914(19)$ \\
O1 & $0.1141(3)$ & $0.43513(17)$ & $0.4285(13)$ & $0.0721(13)$ \\
H1 & 0.148899 & 0.407604 & 0.393280 & $0.108^{*}$ \\
N1 & $0.2784(3)$ & $0.37325(18)$ & $0.4176(13)$ & $0.0605(13)$ \\
C8 & $0.2751(4)$ & $0.4584(2)$ & $0.6892(16)$ & $0.0575(13)$ \\
C1 & $0.4310(5)$ & $0.2352(2)$ & $0.0459(14)$ & $0.0578(12)$ \\
C9 & $0.3293(4)$ & $0.4960(2)$ & $0.875(2)$ & $0.0614(12)$ \\
H9 & 0.397472 & 0.488588 & 0.939016 & $0.074^{*}$ \\
C13 & $0.1701(4)$ & $0.4702(2)$ & $0.6025(17)$ & $0.0564(12)$ \\
C11 & $0.1808(4)$ & $0.5566(2)$ & $0.870(2)$ & $0.0688(15)$ \\
H11 & 0.151047 & 0.589917 & 0.926484 & $0.083^{*}$ \\
C6 & $0.3291(5)$ & $0.2321(2)$ & $0.1458(18)$ & $0.0689(16)$ \\
H6 & 0.293084 & 0.199177 & 0.130244 & $0.083^{*}$ \\
C10 & $0.2824(4)$ & $0.5444(2)$ & $0.9636(16)$ & $0.0618(15)$ \\
O3 & $0.3034(5)$ & $0.6282(2)$ & $1.207(2)$ & $0.120(3)$ \\
N2 & $0.3421(4)$ & $0.5833(2)$ & $1.1596(16)$ & $0.0751(16)$ \\
C7 & $0.3254(4)$ & $0.4086(2)$ & $0.5862(18)$ & $0.0592(12)$ \\
H7 & 0.394439 & 0.402274 & 0.645609 & $0.071^{*}$ \\
C3 & $0.4358(5)$ & $0.3296(2)$ & $0.1943(18)$ & $0.0654(15)$ \\
H3 & 0.472183 & 0.362411 & 0.211171 & $0.079^{*}$ \\
C12 & $0.1245(4)$ & $0.5192(3)$ & $0.6944(17)$ & $0.0655(15)$ \\
H12 & 0.055800 & 0.526809 & 0.636953 & $0.079^{*}$ \\
C2 & $0.4847(4)$ & $0.2839(3)$ & $0.067(2)$ & $0.0679(16)$ \\
H2 & 0.553487 & 0.286056 & -0.003196 & $0.082^{*}$ \\
C4 & $0.3331(4)$ & $0.3266(2)$ & $0.2959(17)$ & $0.0575(15)$ \\
C5 & $0.2798(4)$ & $0.2777(2)$ & $0.2694(15)$ & $0.0660(18)$ \\
H5 & 0.210481 & 0.275532 & 0.335185 & $0.079^{*}$ \\
O2 & $0.4261(4)$ & $0.5694(2)$ & $1.2640(15)$ & $0.0946(19)$ \\
& & & & \\
\end{tabular}

Atomic displacement parameters $\left(\AA^{2}\right)$

\begin{tabular}{lllllll}
\hline & $U^{11}$ & $U^{22}$ & $U^{33}$ & $U^{12}$ & $U^{13}$ & $U^{23}$ \\
\hline I1 & $0.0821(3)$ & $0.0597(3)$ & $0.0656(3)$ & $0.01321(13)$ & $-0.0044(3)$ & $-0.0083(2)$ \\
O1 & $0.0590(19)$ & $0.063(2)$ & $0.094(4)$ & $0.0028(16)$ & $-0.007(2)$ & $-0.007(2)$ \\
N1 & $0.061(2)$ & $0.051(2)$ & $0.069(4)$ & $0.0033(17)$ & $-0.004(2)$ & $0.002(2)$
\end{tabular}




$\begin{array}{lllllll}\text { C8 } & 0.054(3) & 0.052(3) & 0.066(4) & 0.002(2) & 0.007(3) & 0.004(2) \\ \text { C1 } & 0.071(3) & 0.049(3) & 0.054(3) & 0.009(2) & -0.006(3) & 0.002(2) \\ \text { C9 } & 0.054(2) & 0.060(3) & 0.070(4) & 0.0001(18) & -0.001(4) & -0.001(3) \\ \text { C13 } & 0.056(3) & 0.049(3) & 0.064(3) & 0.001(2) & 0.004(3) & 0.003(3) \\ \text { C11 } & 0.067(3) & 0.058(3) & 0.081(4) & 0.007(2) & 0.022(4) & 0.000(4) \\ \text { C6 } & 0.070(3) & 0.054(3) & 0.083(5) & -0.002(2) & -0.006(3) & -0.007(3) \\ \text { C10 } & 0.064(3) & 0.056(3) & 0.066(4) & -0.006(2) & 0.010(2) & -0.001(2) \\ \text { O3 } & 0.111(4) & 0.077(3) & 0.171(8) & 0.001(3) & -0.007(4) & -0.049(4) \\ \text { N2 } & 0.074(3) & 0.066(3) & 0.085(5) & -0.015(2) & 0.014(3) & -0.015(3) \\ \text { C7 } & 0.057(3) & 0.052(3) & 0.068(3) & 0.004(2) & -0.001(3) & 0.008(3) \\ \text { C3 } & 0.068(3) & 0.049(3) & 0.079(4) & -0.004(2) & 0.001(3) & -0.004(2) \\ \text { C12 } & 0.054(3) & 0.061(3) & 0.081(4) & 0.006(2) & 0.003(3) & -0.001(3) \\ \text { C2 } & 0.059(3) & 0.069(4) & 0.075(5) & 0.002(2) & 0.005(3) & 0.003(4) \\ \text { C4 } & 0.062(2) & 0.046(2) & 0.064(5) & 0.0059(19) & -0.005(3) & 0.002(2) \\ \text { C5 } & 0.060(3) & 0.060(3) & 0.078(5) & 0.003(2) & -0.003(3) & -0.005(3) \\ \text { O2 } & 0.072(2) & 0.088(3) & 0.124(6) & -0.012(2) & -0.008(3) & -0.028(3)\end{array}$

Geometric parameters $\left(\AA,{ }^{\circ}\right)$

\begin{tabular}{|c|c|c|c|}
\hline $\mathrm{I} 1-\mathrm{C} 1$ & $2.089(5)$ & $\mathrm{C} 11-\mathrm{H} 11$ & 0.9300 \\
\hline $\mathrm{O} 1-\mathrm{C} 13$ & $1.330(7)$ & $\mathrm{C} 6-\mathrm{C} 5$ & $1.381(8)$ \\
\hline $\mathrm{O} 1-\mathrm{H} 1$ & 0.8200 & C6-H6 & 0.9300 \\
\hline $\mathrm{N} 1-\mathrm{C} 7$ & $1.264(8)$ & $\mathrm{C} 10-\mathrm{N} 2$ & $1.466(8)$ \\
\hline $\mathrm{N} 1-\mathrm{C} 4$ & $1.430(7)$ & $\mathrm{O} 3-\mathrm{N} 2$ & $1.220(8)$ \\
\hline $\mathrm{C} 8-\mathrm{C} 9$ & $1.385(9)$ & $\mathrm{N} 2-\mathrm{O} 2$ & $1.208(8)$ \\
\hline $\mathrm{C} 8-\mathrm{C} 13$ & $1.421(7)$ & $\mathrm{C} 7-\mathrm{H} 7$ & 0.9300 \\
\hline $\mathrm{C} 8-\mathrm{C} 7$ & $1.444(8)$ & $\mathrm{C} 3-\mathrm{C} 4$ & $1.383(8)$ \\
\hline $\mathrm{C} 1-\mathrm{C} 6$ & $1.371(9)$ & $\mathrm{C} 3-\mathrm{C} 2$ & $1.385(10)$ \\
\hline $\mathrm{C} 1-\mathrm{C} 2$ & $1.377(9)$ & $\mathrm{C} 3-\mathrm{H} 3$ & 0.9300 \\
\hline $\mathrm{C} 9-\mathrm{C} 10$ & $1.377(7)$ & $\mathrm{C} 12-\mathrm{H} 12$ & 0.9300 \\
\hline C9- H9 & 0.9300 & $\mathrm{C} 2-\mathrm{H} 2$ & 0.9300 \\
\hline $\mathrm{C} 13-\mathrm{C} 12$ & $1.388(8)$ & $\mathrm{C} 4-\mathrm{C} 5$ & $1.381(8)$ \\
\hline $\mathrm{C} 11-\mathrm{C} 12$ & $1.373(10)$ & $\mathrm{C} 5-\mathrm{H} 5$ & 0.9300 \\
\hline $\mathrm{C} 11-\mathrm{C} 10$ & $1.389(9)$ & & \\
\hline $\mathrm{C} 13-\mathrm{O} 1-\mathrm{H} 1$ & 109.5 & $\mathrm{C} 11-\mathrm{C} 10-\mathrm{N} 2$ & $120.2(5)$ \\
\hline $\mathrm{C} 7-\mathrm{N} 1-\mathrm{C} 4$ & $120.5(5)$ & $\mathrm{O} 2-\mathrm{N} 2-\mathrm{O} 3$ & $123.8(6)$ \\
\hline $\mathrm{C} 9-\mathrm{C} 8-\mathrm{C} 13$ & $118.7(5)$ & $\mathrm{O} 2-\mathrm{N} 2-\mathrm{C} 10$ & $118.8(5)$ \\
\hline $\mathrm{C} 9-\mathrm{C} 8-\mathrm{C} 7$ & $120.1(5)$ & $\mathrm{O} 3-\mathrm{N} 2-\mathrm{C} 10$ & $117.4(6)$ \\
\hline $\mathrm{C} 13-\mathrm{C} 8-\mathrm{C} 7$ & $121.2(5)$ & $\mathrm{N} 1-\mathrm{C} 7-\mathrm{C} 8$ & $121.9(5)$ \\
\hline $\mathrm{C} 6-\mathrm{C} 1-\mathrm{C} 2$ & $120.2(6)$ & $\mathrm{N} 1-\mathrm{C} 7-\mathrm{H} 7$ & 119.1 \\
\hline $\mathrm{C} 6-\mathrm{C} 1-\mathrm{I} 1$ & $120.4(4)$ & $\mathrm{C} 8-\mathrm{C} 7-\mathrm{H} 7$ & 119.1 \\
\hline $\mathrm{C} 2-\mathrm{C} 1-\mathrm{I} 1$ & $119.4(4)$ & $\mathrm{C} 4-\mathrm{C} 3-\mathrm{C} 2$ & $120.2(5)$ \\
\hline $\mathrm{C} 10-\mathrm{C} 9-\mathrm{C} 8$ & $120.0(5)$ & $\mathrm{C} 4-\mathrm{C} 3-\mathrm{H} 3$ & 119.9 \\
\hline $\mathrm{C} 10-\mathrm{C} 9-\mathrm{H} 9$ & 120.0 & $\mathrm{C} 2-\mathrm{C} 3-\mathrm{H} 3$ & 119.9 \\
\hline $\mathrm{C} 8-\mathrm{C} 9-\mathrm{H} 9$ & 120.0 & $\mathrm{C} 11-\mathrm{C} 12-\mathrm{C} 13$ & $120.0(5)$ \\
\hline $\mathrm{O} 1-\mathrm{C} 13-\mathrm{C} 12$ & $118.6(5)$ & $\mathrm{C} 11-\mathrm{C} 12-\mathrm{H} 12$ & 120.0 \\
\hline $\mathrm{O} 1-\mathrm{C} 13-\mathrm{C} 8$ & $121.1(5)$ & $\mathrm{C} 13-\mathrm{C} 12-\mathrm{H} 12$ & 120.0 \\
\hline
\end{tabular}




$\begin{array}{ll}\mathrm{C} 12-\mathrm{C} 13-\mathrm{C} 8 & 120.2(5) \\ \mathrm{C} 12-\mathrm{C} 11-\mathrm{C} 10 & 119.8(5) \\ \mathrm{C} 12-\mathrm{C} 11-\mathrm{H} 11 & 120.1 \\ \mathrm{C} 10-\mathrm{C} 11-\mathrm{H} 11 & 120.1 \\ \mathrm{C} 1-\mathrm{C} 6-\mathrm{C} 5 & 120.1(6) \\ \mathrm{C} 1-\mathrm{C} 6-\mathrm{H} 6 & 119.9 \\ \mathrm{C} 5-\mathrm{C} 6-\mathrm{H} 6 & 121.2(6) \\ \mathrm{C} 9-\mathrm{C} 10-\mathrm{C} 11 & 118.6(5) \\ \mathrm{C} 9-\mathrm{C} 10-\mathrm{N} 2 & \\ & \\ \mathrm{C} 13-\mathrm{C} 8-\mathrm{C} 9-\mathrm{C} 10 & 177.8(7) \\ \mathrm{C} 7-\mathrm{C} 8-\mathrm{C} 9-\mathrm{C} 10 & -179.1(6) \\ \mathrm{C} 9-\mathrm{C} 8-\mathrm{C} 13-\mathrm{O} 1 & 1.3(9) \\ \mathrm{C} 7-\mathrm{C} 8-\mathrm{C} 13-\mathrm{O} 1 & 2.0(9) \\ \mathrm{C} 9-\mathrm{C} 8-\mathrm{C} 13-\mathrm{C} 12 & -177.7(6) \\ \mathrm{C} 7-\mathrm{C} 8-\mathrm{C} 13-\mathrm{C} 12 & -0.2(10) \\ \mathrm{C} 2-\mathrm{C} 1-\mathrm{C} 6-\mathrm{C} 5 & -179.0(5) \\ \mathrm{I} 1-\mathrm{C} 1-\mathrm{C} 6-\mathrm{C} 5 & -0.1(10) \\ \mathrm{C} 8-\mathrm{C} 9-\mathrm{C} 10-\mathrm{C} 11 & -179.9(6) \\ \mathrm{C} 8-\mathrm{C} 9-\mathrm{C} 10-\mathrm{N} 2 & 2.1(11) \\ \mathrm{C} 12-\mathrm{C} 11-\mathrm{C} 10-\mathrm{C} 9 & -178.2(7) \\ \mathrm{C} 12-\mathrm{C} 11-\mathrm{C} 10-\mathrm{N} 2 & -7.4(10) \\ \mathrm{C} 9-\mathrm{C} 10-\mathrm{N} 2-\mathrm{O} 2 & 172.8(7) \\ \mathrm{C} 11-\mathrm{C} 10-\mathrm{N} 2-\mathrm{O} 2 & 172.4(7) \\ \mathrm{C} 9-\mathrm{C} 10-\mathrm{N} 2-\mathrm{O} 3 & -7.4(10) \\ \mathrm{C} 11-\mathrm{C} 10-\mathrm{N} 2-\mathrm{O} 3 & \\ & \end{array}$

$119.8(6)$

120.1

120.1

$119.4(5)$

$118.5(5)$

$122.1(5)$

$120.2(5)$

119.9

119.9

$174.5(6)$

$179.8(6)$

$-0.5(10)$

$-1.9(11)$

$-179.1(7)$

$-0.1(10)$

$0.9(11)$

$179.7(6)$

$-0.7(12)$

$0.0(11)$

$-177.7(7)$

$146.2(7)$

$-36.1(10)$

$-0.6(10)$

$0.7(10)$

$178.5(6)$

Hydrogen-bond geometry $\left(A,{ }^{\circ}\right)$

\begin{tabular}{lllll}
\hline$D-\mathrm{H} \cdots A$ & $D-\mathrm{H}$ & $\mathrm{H} \cdots A$ & $D \cdots A$ & $D-\mathrm{H} \cdots A$ \\
\hline $\mathrm{O} 1-\mathrm{H} 1 \cdots \mathrm{N} 1$ & 0.82 & 1.86 & $2.591(6)$ & 148 \\
$\mathrm{C} 7-\mathrm{H} 7 \cdots \mathrm{O} 2^{\mathrm{i}}$ & 0.93 & 2.45 & $3.309(8)$ & 154 \\
\hline
\end{tabular}

Symmetry code: (i) $-x+1,-y+1, z-1 / 2$. 